\title{
Smooth Optimization Approach for Sparse Covariance Selection
}

\author{
Zhaosong Lu* \\ July 3, 2007 (Revised: January, August, September 2008)
}

\begin{abstract}
In this paper we first study a smooth optimization approach for solving a class of non-smooth strictly concave maximization problems whose objective functions admit smooth convex minimization reformulations. In particular, we apply Nesterov's smooth optimization technique [19, 21] to their dual counterparts that are smooth convex problems. It is shown that the resulting approach has $\mathcal{O}(1 / \sqrt{\epsilon})$ iteration complexity for finding an $\epsilon$-optimal solution to both primal and dual problems. We then discuss the application of this approach to sparse covariance selection that is approximately solved as a $l_{1}$-norm penalized maximum likelihood estimation problem, and also propose a variant of this approach which has substantially outperformed the latter one in our computational experiments. We finally compare the performance of these approaches with other first-order methods, namely, Nesterov's $\mathcal{O}(1 / \epsilon)$ smooth approximation scheme and block-coordinate descent method studied in [9, 15] for sparse covariance selection on a set of randomly generated instances. It shows that our smooth optimization approach substantially outperforms the first method above, and moreover, its variant substantially outperforms both methods above.
\end{abstract}

Key words: Covariance selection, non-smooth strictly concave maximization, smooth minimization

AMS 2000 subject classification: 90C22, 90C25, 90C47, 65K05, 62J10

\section{Introduction}

In [19, 21], Nesterov proposed an efficient smooth optimization method for solving convex programming problems of the form

$$
\min \{f(u): u \in U\},
$$

\footnotetext{
*Department of Mathematics, Simon Fraser University, Burnaby, BC, V5A 1S6, Canada. (email: zhaosong@sfu.ca). This author was supported in part by SFU President's Research Grant and NSERC Discovery Grant.
} 
where $f$ is a convex function with Lipschitz continuous gradient, and $U$ is a closed convex set. It is shown that his method has $\mathcal{O}(1 / \sqrt{\epsilon})$ iteration complexity bound, where $\epsilon>0$ is the absolute precision of the final objective function value. A proximal-point-type algorithm for (1) having the same complexity as above has also been proposed more recently by Auslender and Teboulle [2].

Motivated by [9], we are particularly interested in studying the use of smooth optimization approach for solving a class of non-smooth strictly concave maximization problems whose objective functions admit smooth convex minimization reformulations in this paper. Our key idea is to apply Nesterov's smooth optimization technique [19, 21] to their dual counterparts that are smooth convex problems. It is shown that the resulting approach has $\mathcal{O}(1 / \sqrt{\epsilon})$ iteration complexity for finding an $\epsilon$-optimal solution to both primal and dual problems.

One interesting application of the above approach is for sparse covariance selection. Given a set of random variables with Gaussian distribution for which the true covariance matrix is unknown, covariance selection is a procedure used to estimate true covariance from a sample covariance matrix by maximizing its likelihood while imposing a certain sparsity on the inverse of the covariance estimation (e.g., see [11]). Therefore, it can be applied to determine a robust estimate of the true variance matrix, and simultaneously discover the sparse structure in the underlying model. Despite its popularity in numerous real-world applications (e.g., see [3, 9, 25] and the references therein), sparse covariance selection itself is a challenging NP-hard combinatorial optimization problem. By an argument that is often used in regression techniques such as LASSO [23], Yuan and Lin [25] and d'Aspremont et al. [9] (see also [3]) showed that it can be approximately solved as a $l_{1}$-norm penalized maximum likelihood estimation problem. Moreover, the authors of [9] studied two efficient first-order methods for solving this problem, that is, Nesterov's smooth approximation scheme and block-coordinate descent (BCD) method. It was shown in [9] that their first method has $\mathcal{O}(1 / \epsilon)$ iteration complexity for finding an $\epsilon$-optimal solution. For their second method, each iterate requires solving a box constrained quadratic programming, and it has a local linear convergence rate. However, its global iteration complexity for finding an $\epsilon$-optimal solution is theoretically unknown. After the first release of our paper, Friedman et al. [15] studied a slight variant of the BCD method proposed in 9. At each iteration of their method, a coordinate descent approach is applied to solve a lasso ( $l_{1}$-regularized) least-squares problem, which is the dual of the box constrained quadratic programming appearing in the BCD method [9]. In contrast with these methods, the smooth optimization approach proposed in this paper has a more attractive iteration complexity that is $\mathcal{O}(1 / \sqrt{\epsilon})$ for finding an $\epsilon$-optimal solution. In addition, we propose a variant of the smooth optimization approach which has substantially outperformed the latter one in our computational experiments. We also compare the performance of our approaches with their methods for sparse covariance selection on a set of randomly generated instances. It shows that our smooth optimization approach substantially outperforms their first method above (i.e., Nesterov's smooth approximation scheme), and moreover, its variant substantially outperforms their methods [9, 15] mentioned above.

The paper is organized as follows. In Section 2, we introduce a class of non-smooth concave maximization problems in which we are interested, and propose a smooth optimization 
approach to them. In Section 3, we briefly introduce sparse covariance selection, and show that it can be approximately solved as a $l_{1}$-norm penalized maximum likelihood estimation problem. We also discuss the application of the smooth optimization approach for solving this problem, and propose a variant of this approach. In Section 4, we compare the performance of our smooth optimization approach and its variant with two other first-order methods studied in [9, 15] for sparse covariance selection on a set of randomly generated instances. Finally, we present some concluding remarks in Section 5 .

\section{$1.1 \quad$ Notation}

In this paper, all vector spaces are assumed to be finite dimensional. The space of symmetric $n \times n$ matrices will be denoted by $\mathcal{S}^{n}$. If $X \in \mathcal{S}^{n}$ is positive semidefinite, we write $X \succeq 0$. Also, we write $X \preceq Y$ to mean $Y-X \succeq 0$. The cone of positive semidefinite (resp., definite) matrices is denoted by $\mathcal{S}_{+}^{n}$ (resp., $\mathcal{S}_{++}^{n}$ ). Given matrices $X$ and $Y$ in $\Re^{p \times q}$, the standard inner product is defined by $\langle X, Y\rangle:=\operatorname{Tr}\left(X Y^{T}\right)$, where $\operatorname{Tr}(\cdot)$ denotes the trace of a matrix. $\|\cdot\|$ denotes the Euclidean norm and its associated operator norm unless it is explicitly stated

otherwise. The Frobenius norm of a real matrix $X$ is defined as $\|X\|_{F}:=\sqrt{\operatorname{Tr}\left(X X^{T}\right)}$. We denote by $e$ the vector of all ones, and by $I$ the identity matrix. Their dimensions should be clear from the context. For a real matrix $X$, we denote by $\operatorname{Card}(X)$ the cardinality of $X$, that is, the number of nonzero entries of $X$, and denote by $|X|$ the absolute value of $X$, that is, $|X|_{i j}=\left|X_{i j}\right|$ for all $i, j$. The determinant and the minimal (resp., maximal) eigenvalue of a real symmetric matrix $X$ are denoted by $\operatorname{det} X$ and $\lambda_{\min }(X)$ (resp., $\lambda_{\max }(X)$ ), respectively. For a $n$-dimensional vector $w, \operatorname{diag}(w)$ denote the diagonal matrix whose $i$-th diagonal element is $w_{i}$ for $i=1, \ldots, n$. We denote by $\mathcal{Z}_{+}$the set of all nonnegative integers.

Let the space $\mathcal{F}$ be endowed with an arbitrary norm $\|\cdot\|$. The dual space of $\mathcal{F}$, denoted by $\mathcal{F}^{*}$, is the normed real vector space consisting of all linear functionals of $s: \mathcal{F} \rightarrow \Re$, endowed with the dual norm $\|\cdot\|^{*}$ defined as

$$
\|s\|^{*}:=\max _{u}\{\langle s, u\rangle:\|u\| \leq 1\}, \quad \forall s \in \mathcal{F}^{*},
$$

where $\langle s, u\rangle:=s(u)$ is the value of the linear functional $s$ at $u$. Finally, given an operator $\mathcal{A}: \mathcal{F} \rightarrow \mathcal{F}^{*}$, we define

$$
\mathcal{A}[H, H]:=\langle\mathcal{A} H, H\rangle
$$

for any $H \in \mathcal{F}$.

\section{Smooth optimization approach}

In this section, we consider a class of concave non-smooth maximization problems:

$$
\max _{x \in X}\left\{g(x):=\min _{u \in U} \phi(x, u)\right\}
$$

where $X$ and $U$ are nonempty convex compact sets in finite-dimensional real vector spaces $\mathcal{E}$ and $\mathcal{F}$, respectively, and $\phi(x, u): X \times U \rightarrow \Re$ is a continuous function which is strictly 
concave in $x \in X$ for every fixed $u \in U$, and convex differentiable in $u \in U$ for every fixed $x \in X$. Therefore, for any $u \in U$, the function

$$
f(u):=\max _{x \in X} \phi(x, u)
$$

is well-defined. We also easily conclude that $f(u)$ is convex differentiable on $U$, and its gradient is given by

$$
\nabla f(u)=\nabla_{u} \phi(x(u), u), \quad \forall u \in U,
$$

where $x(u)$ denotes the unique solution of (3) .

Let the space $\mathcal{F}$ be endowed with an arbitrary norm $\|\cdot\|$. We further assume that $\nabla f(u)$ is Lipschitz continuous on $U$ with respect to $\|\cdot\|$, i.e., there exists some $L>0$ such that

$$
\|\nabla f(u)-\nabla f(\tilde{u})\|^{*} \leq L\|u-\tilde{u}\|, \quad \forall u, \tilde{u} \in U .
$$

Under the above assumptions, we easily observe that: i) problem (2) and its dual, that is,

$$
\min _{u}\{f(u): u \in U\}
$$

are both solvable and have the same optimal value; and ii) the dual problem (5) can be suitably solved by Nesterov's smooth minimization approach [19, 21].

Denote by $d(u)$ a prox-function of the set $U$. We assume that $d(u)$ is continuous and strongly convex on $U$ with modulus $\sigma>0$. Let $u_{0}$ be the center of the set $U$ defined as

$$
u_{0}=\arg \min \{d(u): u \in U\} .
$$

Without loss of generality assume that $d\left(u_{0}\right)=0$. We now describe Nesterov's smooth minimization approach [19, 21] for solving the dual problem (5), and we will show that it simultaneously solves the non-smooth concave maximization problem (2).

\section{Smooth Minimization Algorithm:}

Let $u_{0} \in U$ be given in (6) . Set $x_{-1}=0$ and $k=0$.

1) Compute $\nabla f\left(u_{k}\right)$ and $x\left(u_{k}\right)$. Set $x_{k}=\frac{k}{k+2} x_{k-1}+\frac{2}{k+2} x\left(u_{k}\right)$.

2) Find $u_{k}^{s d} \in \operatorname{Argmin}\left\{\left\langle\nabla f\left(u_{k}\right), u-u_{k}\right\rangle+\frac{L}{2}\left\|u-u_{k}\right\|^{2}: u \in U\right\}$.

3) Find $u_{k}^{a g}=\operatorname{argmin}\left\{\frac{L}{\sigma} d(u)+\sum_{i=0}^{k} \frac{i+1}{2}\left[f\left(u_{i}\right)+\left\langle\nabla f\left(u_{i}\right), u-u_{i}\right\rangle\right]: u \in U\right\}$.

4) Set $u_{k+1}=\frac{2}{k+3} u_{k}^{a g}+\frac{k+1}{k+3} u_{k}^{s d}$.

5) Set $k \leftarrow k+1$ and go to step 1 ).

\section{end}

The following property of the above algorithm is established in Theorem 2 of Nesterov 21]. 
Theorem 2.1 Let the sequence $\left\{\left(u_{k}, u_{k}^{s d}\right)\right\}_{k=0}^{\infty} \subseteq U \times U$ be generated by the Smooth Minimization Algorithm. Then for any $k \geq 0$ we have

$$
\frac{(k+1)(k+2)}{4} f\left(u_{k}^{s d}\right) \leq \min \left\{\frac{L}{\sigma} d(u)+\sum_{i=0}^{k} \frac{i+1}{2}\left[f\left(u_{i}\right)+\left\langle\nabla f\left(u_{i}\right), u-u_{i}\right\rangle\right]: u \in U\right\} .
$$

We are ready to establish the main convergence result of the Smooth Minimization Algorithm for solving the non-smooth concave maximization problem (2) and its dual (5). Its proof is a generalization of the one given in a more special context in [21].

Theorem 2.2 After $k$ iterations, the Smooth Minimization Algorithm generates a pair of approximate solutions $\left(u_{k}^{s d}, x_{k}\right)$ to problem (2) and its dual (5), respectively, which satisfy the following inequality:

$$
0 \leq f\left(u_{k}^{s d}\right)-g\left(x_{k}\right) \leq \frac{4 L D}{\sigma(k+1)(k+2)} .
$$

Thus if the termination criterion $f\left(u_{k}^{s d}\right)-g\left(x_{k}\right) \leq \epsilon$ is applied, the iteration complexity of finding an e-optimal solution to problem (2) and its dual (5) by the Smooth Minimization Algorithm does not exceed $2 \sqrt{\frac{L D}{\sigma \epsilon}}$, where

$$
D=\max \{d(u): u \in U\}
$$

Proof. In view of (3), (41) and the notation $x(u)$, we have

$$
f\left(u_{i}\right)+\left\langle\nabla f\left(u_{i}\right), u-u_{i}\right\rangle=\phi\left(x\left(u_{i}\right), u_{i}\right)+\left\langle\nabla_{u} \phi\left(x\left(u_{i}\right), u_{i}\right), u-u_{i}\right\rangle .
$$

Invoking the fact that the function $\phi(x, \cdot)$ is convex on $U$ for every fixed $x \in X$, we obtain

$$
\phi\left(x\left(u_{i}\right), u_{i}\right)+\left\langle\nabla_{u} \phi\left(x\left(u_{i}\right), u_{i}\right), u-u_{i}\right\rangle \leq \phi\left(x\left(u_{i}\right), u\right)
$$

Notice that $x_{-1}=0$, and $x_{k}=\frac{k}{k+2} x_{k-1}+\frac{2}{k+2} x\left(u_{k}\right)$ for any $k \geq 0$, which imply

$$
x_{k}=\sum_{i=0}^{k} \frac{2(i+1)}{(k+1)(k+2)} x\left(u_{i}\right) .
$$

Using (10), (11), (12) and the fact that the function $\phi(\cdot, u)$ is concave on $X$ for every fixed $u \in U$, we have

$$
\begin{aligned}
\sum_{i=0}^{k}(i+1)\left[f\left(u_{i}\right)+\left\langle\nabla f\left(u_{i}\right), u-u_{i}\right\rangle\right] & \leq \sum_{i=0}^{k}(i+1) \phi\left(x\left(u_{i}\right), u\right) \\
& \leq \frac{1}{2}(k+1)(k+2) \phi\left(x_{k}, u\right)
\end{aligned}
$$


for all $u \in U$. It follows from this relation, (17), (9) and (2) that

$$
\begin{aligned}
f\left(u_{k}^{s d}\right) & \leq \frac{4 L D}{\sigma(k+1)(k+2)}+\min _{u}\left\{\sum_{i=0}^{k} \frac{2(i+1)}{(k+1)(k+2)}\left[f\left(u_{i}\right)+\left\langle\nabla f\left(u_{i}\right), u-u_{i}\right\rangle\right]: u \in U\right\} \\
& \leq \frac{4 L D}{\sigma(k+1)(k+2)}+\min _{u \in U} \phi\left(x_{k}, u\right)=\frac{4 L D}{\sigma(k+1)(k+2)}+g\left(x_{k}\right),
\end{aligned}
$$

and hence the inequality (8) holds. The remaining conclusion directly follows from (8) .

Remark. We shall mention that Nesterov [20] developed the excessive gap technique for solving problem (2) and its dual (5) in a special context, which enjoys the same iteration complexity as the Smooth Minimization Algorithm described above. In addition, it is not hard to observe that the technique proposed in [20] can be extended to solve problem (2) and its dual (5) in the aforementioned general framework provided that the subproblem

$$
\min _{u \in U} \phi(x, u)+\mu d(u)
$$

can be suitably solved for any given $\mu>0$ and $x \in X$. The computation of each iterate of Nesterov's excessive gap technique [20] is similar to that of the Smooth Minimization Algorithm except that the former method requires solving a prox subproblem in the form of (13), but the latter one needs to solve the prox subproblem described in step 3) above. When the function $\phi(x, \cdot)$ is affine for every fixed $x \in X$, these two prox subproblems have the same form, and thus the computational cost of Nesterov's excessive gap technique [20] is almost same as that of the Smooth Minimization Algorithm; however, for a more general function $\phi(\cdot, \cdot)$, the computational cost of the former method can be more expensive than that of the latter method.

The following results will be used to develop a variant of the Smooth Minimization Algorithm for sparse covariance selection in Subsection 3.4

Lemma 2.3 Problem (2) has a unique optimal solution, denoted by $x^{*}$. Moreover, for any $u^{*} \in \operatorname{Argmin}\{f(u): u \in U\}$, we have

$$
x^{*}=\arg \max _{x \in X} \phi\left(x, u^{*}\right) .
$$

Proof. We clearly know that problem (2) has an optimal solution. To prove its uniqueness, it suffices to show that $g(x)$ is strictly concave on $X$. Indeed, since $X \times U$ is a convex compact set and $\phi(x, u)$ is continuous on $X \times U$, it follows that for any $t \in(0,1), x^{1} \neq x^{2} \in X$, there exists some $\tilde{u} \in U$ such that

$$
\phi\left(t x^{1}+(1-t) x^{2}, \tilde{u}\right)=\min _{u \in U} \phi\left(t x^{1}+(1-t) x^{2}, u\right) .
$$

Recall that $\phi(\cdot, u)$ is strictly concave on $X$ for every fixed $u \in U$. Therefore, we have

$$
\begin{aligned}
\phi\left(t x^{1}+(1-t) x^{2}, \tilde{u}\right) & >t \phi\left(x^{1}, \tilde{u}\right)+(1-t) \phi\left(x^{2}, \tilde{u}\right), \\
& \geq t \min _{u \in U} \phi\left(x^{1}, u\right)+(1-t) \min _{u \in U} \phi\left(x^{2}, u\right),
\end{aligned}
$$


which together with (2) implies that

$$
g\left(t x^{1}+(1-t) x^{2}\right)>\operatorname{tg}\left(x^{1}\right)+(1-t) g\left(x^{2}\right)
$$

for any $t \in(0,1), x^{1} \neq x^{2} \in X$, and hence, $g(x)$ is strictly concave on $X$ as desired.

Note that $x^{*}$ is the optimal solution of problem (2). We clearly know that for any $u^{*} \in$ $\operatorname{Argmin}\{f(u): u \in U\},\left(u^{*}, x^{*}\right)$ is a saddle point for problem (2), that is,

$$
\phi\left(x^{*}, u\right) \geq \phi\left(x^{*}, u^{*}\right) \geq \phi\left(x, u^{*}\right), \quad \forall(x, u) \in X \times U,
$$

and hence, we have

$$
x^{*} \in \operatorname{Arg} \max _{x \in X} \phi\left(x, u^{*}\right) .
$$

It together with the fact that $\phi\left(\cdot, u^{*}\right)$ is strictly concave on $X$, immediately yields (14).

Theorem 2.4 Let $x^{*}$ be the unique optimal solution of (2), and $f^{*}$ be the optimal value of problems (2) and (5). Assume that the sequences $\left\{u_{k}\right\}_{k=0}^{\infty}$ and $\left\{x\left(u_{k}\right)\right\}_{k=0}^{\infty}$ are generated by the Smooth Minimization Algorithm. Then the following statements hold:

1) $f\left(u_{k}\right) \rightarrow f^{*}, x\left(u_{k}\right) \rightarrow x^{*}$ as $k \rightarrow \infty$;

2) $f\left(u_{k}\right)-g\left(x\left(u_{k}\right)\right) \rightarrow 0$ as $k \rightarrow \infty$.

Proof. Recall from the Smooth Minimization Algorithm that

$$
u_{k+1}=\left(2 u_{k}^{a g}+(k+1) u_{k}^{s d}\right) /(k+3), \quad \forall k \geq 0 .
$$

Since $u_{k}^{s d}, u_{k}^{a g} \in U$ for $\forall k \geq 0$, and $U$ is a compact set, we have $u_{k+1}-u_{k}^{s d} \rightarrow 0$ as $k \rightarrow \infty$. Notice that $f(u)$ is continuous on the compact set $U$, and hence, it is uniformly continuous on $U$. Then we further have $f\left(u_{k+1}\right)-f\left(u_{k}^{s d}\right) \rightarrow 0$ as $k \rightarrow \infty$. Also, it follows from Theorem 2.2 that $f\left(u_{k}^{s d}\right) \rightarrow f^{*}$ as $k \rightarrow \infty$. Therefore, we conclude that $f\left(u_{k}\right) \rightarrow f^{*}$ as $k \rightarrow \infty$.

Note that $X$ is a compact set, and $x\left(u_{k}\right) \subseteq X$ for $\forall k \geq 0$. To prove that $x\left(u_{k}\right) \rightarrow x^{*}$ as $k \rightarrow \infty$, it suffices to show that every convergent subsequence of $\left\{x\left(u_{k}\right)\right\}_{k=0}^{\infty}$ converges to $x^{*}$ as $k \rightarrow \infty$. Indeed, assume that $\left\{x\left(u_{n_{k}}\right)\right\}_{k=0}^{\infty}$ is an arbitrary convergent subsequence, and $x\left(u_{n_{k}}\right) \rightarrow \tilde{x}^{*}$ as $k \rightarrow \infty$ for some $\tilde{x}^{*} \in X$. Without loss generality, assume that the sequence $\left\{u_{n_{k}}\right\}_{k=0}^{\infty} \rightarrow \tilde{u}^{*}$ as $k \rightarrow \infty$ for some $\tilde{u}^{*} \in U$ (otherwise, one can consider any convergent subsequence of $\left.\left\{u_{n_{k}}\right\}_{k=0}^{\infty}\right)$. Using the result that $f\left(u_{k}\right) \rightarrow f^{*}$, we obtain that

$$
\phi\left(x\left(u_{n_{k}}\right), u_{n_{k}}\right)=f\left(u_{n_{k}}\right) \rightarrow f^{*}, \quad \text { as } k \rightarrow \infty .
$$

Upon letting $k \rightarrow \infty$ and using the continuity of $\phi(\cdot, \cdot)$, we have $\phi\left(\tilde{x}^{*}, \tilde{u}^{*}\right)=f\left(\tilde{u}^{*}\right)=f^{*}$. Hence, it follows that

$$
\tilde{u}^{*} \in \operatorname{Arg} \min _{u \in U} f(u), \quad \tilde{x}^{*}=\arg \max _{x \in X} \phi\left(x, \tilde{u}^{*}\right),
$$

which together with Lemma 2.3 implies that $\tilde{x}^{*}=x^{*}$. Hence as desired, $x\left(u_{n_{k}}\right) \rightarrow x^{*}$ as $k \rightarrow \infty$.

As shown in Lemma 2.3, the function $g(x)$ is continuous on $X$. This result together with statement 1) immediately implies that statement 2) holds. 


\section{Sparse covariance selection}

In this section, we discuss the application of the smooth optimization approach proposed in Section 2 to sparse covariance selection. More specifically, we briefly introduce sparse covariance selection in Subsection 3.1, and show that it can be approximately solved as a $l_{1}$-norm penalized maximum likelihood estimation problem in Subsection 3.2. In Subsection 3.3, We address some implementation details of the smooth optimization approach for solving this problem, and propose a variant of this approach in Subsection 3.4.

\subsection{Introduction of sparse covariance selection}

In this subsection, we briefly introduce sparse covariance selection. For more details, see d'Aspremont et al. [9] and the references therein.

Given $n$ variables with a Gaussian distribution $\mathcal{N}(0, C)$ for which the true covariance matrix $C$ is unknown, we are interested in estimating $C$ from a sample covariance matrix $\Sigma$ by maximizing its likelihood while imposing a certain number of components in the inverse of the estimation of $C$ to zero. This problem is commonly known as sparse covariance selection (see [11]). Since zeros in the inverse of covariance matrix correspond to conditional independence in the model, sparse covariance selection can be used to determine a robust estimate of the covariance matrix, and simultaneously discover the sparse structure in the underlying graphical model.

Several approaches have been proposed for sparse covariance selection in literature. For example, Bilmes [4] proposed a method based on choosing statistical dependencies according to conditional mutual information computed from training data. The recent works [18, 13] involve identifying the Gaussian graphical models that are best supported by the data and any available prior information on the covariance matrix. Given a sample covariance matrix $\Sigma \in \mathcal{S}_{+}^{n}$, d'Aspremont et al. 9] recently formulated sparse covariance selection as the following estimation problem:

$$
\begin{array}{ll}
\max _{X} & \log \operatorname{det} X-\langle\Sigma, X\rangle-\rho \operatorname{Card}(X) \\
\text { s.t. } & \tilde{\alpha} I \preceq X \preceq \tilde{\beta} I,
\end{array}
$$

where $\rho>0$ is a parameter controlling the trade-off between likelihood and cardinality, and $0 \leq \tilde{\alpha}<\tilde{\beta} \leq \infty$ are the fixed bounds on the eigenvalues of the solution. For some specific choices of $\rho$, the formulation (15) has been used for model selection in [1, 5], and applied to speech recognition and gene network analysis (see [4, 12]).

Note that the estimation problem (15) itself is a NP-hard combinatorial problem because of the penalty term $\operatorname{Card}(X)$. To overcome the computational difficulty, d'Aspremont et al. [9] used an argument that is often used in regression techniques (e.g., see [23, 6, 14]), where sparsity of the solution is concerned, to relax $\operatorname{Card}(X)$ to $e^{T}|X| e$, and obtained the following $l_{1}$-norm penalized maximum likelihood estimation problem:

$$
\begin{array}{ll}
\max _{X} & \log \operatorname{det} X-\langle\Sigma, X\rangle-\rho e^{T}|X| e \\
\text { s.t. } & \tilde{\alpha} I \preceq X \preceq \tilde{\beta} I,
\end{array}
$$


Recently, Yuan and Lin [25] proposed a similar estimation problem for sparse covariance selection given as follows:

$$
\begin{array}{ll}
\max _{X} & \log \operatorname{det} X-\langle\Sigma, X\rangle-\rho \sum_{i \neq j}\left|X_{i j}\right| \\
\text { s.t. } & \tilde{\alpha} I \preceq X \preceq \tilde{\beta} I,
\end{array}
$$

with $\tilde{\alpha}=0$ and $\tilde{\beta}=\infty$. They showed that problem (17) can be suitably solved by the interior point algorithm developed in Vandenberghe et al. 24]. A few other approaches have also been studied for sparse covariance selection by solving some related maximum likelihood estimation problems in literature. For example, Huang et al. [17] proposed an iterative (heuristic) algorithm to minimize a nonconvex penalized likelihood. Dahl et al. [8, 7] applied Newton method, coordinate steepest descent method, and conjugate gradient method for the problems for which the conditional independence structure is partially known.

As shown in d'Aspremont et al. [9] (see also [3]), and Yuan and Lin [25], the $l_{1}$-norm penalized maximum likelihood estimation problems (16) and (17) are capable of discovering effectively the sparse structure, or equivalently, the conditional independence in the underlying graphical model. Also, it is not hard to see that the estimation problem (17) becomes a special case of problem (16) if replacing $\Sigma$ by $\Sigma+\rho I$ in (17). For these reasons, we focus on problem (16) only for the remaining paper.

\subsection{Non-smooth strictly concave maximization reformulation}

In this subsection, we show that problem (16) can be reformulated as a non-smooth strictly concave maximization problem of the form (2).

Recall from Subsection 3.1 that $\Sigma \in \mathcal{S}_{+}^{n}$, and keep in mind that the notations $|\cdot|,\|\cdot\|$ and $\|\cdot\|_{F}$ are defined in Subsection 1.1. We first provide some tighter bounds on the optimal solution of problem (16) for the case where $\tilde{\alpha}=0$ and $\tilde{\beta}=\infty$.

Proposition 3.1 Assume that $\tilde{\alpha}=0$ and $\tilde{\beta}=\infty$. Let $X^{*} \in \mathcal{S}_{++}^{n}$ be the unique optimal solution of problem (16). Then we have $\alpha I \preceq X^{*} \preceq \beta I$, where

$$
\alpha=\frac{1}{\|\Sigma\|+n \rho}, \quad \beta=\min \left\{\frac{n-\alpha \operatorname{Tr}(\Sigma)}{\rho}, \eta\right\}
$$

with

$$
\eta= \begin{cases}\min \left\{e^{T}\left|\Sigma^{-1}\right| e,(n-\rho \sqrt{n} \alpha) \| \Sigma^{-1} \mid-(n-1) \alpha\right\}, & \text { if } \Sigma \text { is invertible } \\ 2 e^{T}\left|\left(\Sigma+\frac{\rho}{2} I\right)^{-1}\right| e-\operatorname{Tr}\left(\left(\Sigma+\frac{\rho}{2} I\right)^{-1}\right), & \text { otherwise. }\end{cases}
$$

Proof. Let

$$
\mathcal{U}:=\left\{U \in \mathcal{S}^{n}:\left|U_{i j}\right| \leq 1, \forall i j\right\}
$$

and

$$
L(X, U)=\log \operatorname{det} X-\langle\Sigma+\rho U, X\rangle, \quad \forall(X, U) \in \mathcal{S}_{++}^{n} \times \mathcal{U}
$$


Note that $X^{*} \in \mathcal{S}_{++}^{n}$ is the optimal solution of problem (16). It can be easily shown that there exists some $U^{*} \in \mathcal{U}$ such that $\left(X^{*}, U^{*}\right)$ is a saddle point of $L(\cdot, \cdot)$ on $\mathcal{S}_{++}^{n} \times \mathcal{U}$, that is,

$$
X^{*}=\arg \min _{X \in \mathcal{S}_{++}^{n}} L\left(X, U^{*}\right), \quad U^{*} \in \operatorname{Arg} \min _{U \in \mathcal{U}} L\left(X^{*}, U\right) .
$$

The above relations along with (19) and (20) immediately yield

$$
X^{*}\left(\Sigma+\rho U^{*}\right)=I, \quad\left\langle X^{*}, U^{*}\right\rangle=e^{T}\left|X^{*}\right| e .
$$

Hence, we have

$$
X^{*}=\left(\Sigma+\rho U^{*}\right)^{-1} \succeq \frac{1}{\|\Sigma\|+\rho\left\|U^{*}\right\|} I,
$$

which together with (19) and the fact $U^{*} \in \mathcal{U}$, implies that $X^{*} \succeq \frac{1}{\|\Sigma\|+n \rho} I$. Thus as desired, $X^{*} \succeq \alpha I$, where $\alpha$ is given in (18).

We next bound $X^{*}$ from above. In view of (21), we have

$$
\left\langle X^{*}, \Sigma\right\rangle+\rho e^{T}\left|X^{*}\right| e=n,
$$

which together with the relation $X^{*} \succeq \alpha I$ implies that

$$
e^{T}\left|X^{*}\right| e \leq \frac{n-\alpha \operatorname{Tr}(\Sigma)}{\rho} .
$$

Now let $X(t):=(\Sigma+t \rho I)^{-1}$ for $t \in(0,1)$. By concavity of $\log \operatorname{det}(\cdot)$, one can easily see that $X(t)$ maximizes the function $\log \operatorname{det}(\cdot)-\langle\Sigma+t \rho I, \cdot\rangle$ over $\mathcal{S}_{++}^{n}$. Using this observation and the definition of $X^{*}$, we can have

$$
\begin{aligned}
\log \operatorname{det} X^{*}-\left\langle\Sigma+t \rho I, X^{*}\right\rangle & \leq \log \operatorname{det} X(t)-\langle\Sigma+t \rho I, X(t)\rangle, \\
\log \operatorname{det} X(t)-\langle\Sigma, X(t)\rangle-\rho e^{T}|X(t)| e & \leq \log \operatorname{det} X^{*}-\left\langle\Sigma, X^{*}\right\rangle-\rho e^{T}\left|X^{*}\right| e .
\end{aligned}
$$

Adding the above two inequalities upon some algebraic simplification, we obtain that

$$
e^{T}\left|X^{*}\right| e-t \operatorname{Tr}\left(X^{*}\right) \leq e^{T}|X(t)| e-t \operatorname{Tr}(X(t)),
$$

and hence,

$$
e^{T}\left|X^{*}\right| e \leq \frac{e^{T}|X(t)| e-t \operatorname{Tr}(X(t))}{1-t}, \quad \forall t \in(0,1)
$$

If $\Sigma$ is invertible, upon letting $t \downarrow 0$ on both sides of (24), we have

$$
e^{T}\left|X^{*}\right| e \leq e^{T}\left|\Sigma^{-1}\right| e .
$$

Otherwise, letting $t=1 / 2$ in (24), we obtain

$$
e^{T}\left|X^{*}\right| e \leq 2 e^{T}\left|\left(\Sigma+\frac{\rho}{2} I\right)^{-1}\right| e-\operatorname{Tr}\left(\left(\Sigma+\frac{\rho}{2} I\right)^{-1}\right) .
$$


Combining the above two inequalities and (23), we have

$$
\left\|X^{*}\right\| \leq\left\|X^{*}\right\|_{F} \leq e^{T}\left|X^{*}\right| e \leq \min \left\{\frac{n-\alpha \operatorname{Tr}(\Sigma)}{\rho}, \gamma\right\} \text {, }
$$

where

$$
\gamma= \begin{cases}e^{T}\left|\Sigma^{-1}\right| e, & \text { if } \Sigma \text { is invertible } \\ 2 e^{T}\left|\left(\Sigma+\frac{\rho}{2} I\right)^{-1}\right| e-\operatorname{Tr}\left(\left(\Sigma+\frac{\rho}{2} I\right)^{-1}\right), & \text { otherwise. }\end{cases}
$$

Further, using the relation $X^{*} \succeq \alpha I$, we obtain that

$$
e^{T}\left|X^{*}\right| e \geq\left\|X^{*}\right\|_{F} \geq \sqrt{n} \alpha
$$

which together with (22) implies that

$$
\operatorname{Tr}\left(X^{*} \Sigma\right) \leq n-\rho \sqrt{n} \alpha
$$

This inequality along with the relation $X^{*} \succeq \alpha I$ yields

$$
\lambda_{\min }(\Sigma)\left((n-1) \alpha+\left\|X^{*}\right\|\right) \leq \operatorname{Tr}\left(X^{*} \Sigma\right) \leq n-\rho \sqrt{n} \alpha .
$$

Hence if $\Sigma$ is invertible, we further have

$$
\left\|X^{*}\right\| \leq(n-\rho \sqrt{n} \alpha)\left\|\Sigma^{-1}\right\|-(n-1) \alpha .
$$

This together with (25) implies that $X^{*} \preceq \beta I$, where $\beta$ is given in (18).

Remark. Some bounds on $X^{*}$ were also derived in d'Aspremont et al. [9]. In contrast with their bounds, our bounds given in (18) are tighter. Moreover, our approach for deriving the above bounds can be generalized to handle the case where $\tilde{\alpha}>0$ and $\tilde{\beta}=\infty$, but their approach cannot. Indeed, if $\tilde{\alpha}>0$ and $\tilde{\beta}=\infty$, we can set $\alpha=\tilde{\alpha}$, and replace the above $X(t)$ by the optimal solution of

$$
\begin{array}{ll}
\max _{X} & \log \operatorname{det} X-\langle\Sigma+\rho I, X\rangle \\
\text { s.t. } & \tilde{\alpha} I \preceq X,
\end{array}
$$

which has a closed-form expression. By following a similar derivation as above, one can obtain a positive scalar $\beta$ such that $X^{*} \preceq \beta I$. In addition, for the case where $\tilde{\alpha}=0$ and $0<\tilde{\beta}<\infty$, one can set $\beta=\tilde{\beta}$ and easily show that $X^{*} \geq \alpha I$, where $\alpha=\beta e^{-\beta(\operatorname{Tr}(\Sigma)+n \rho)}$.

From the above discussion, we conclude that problem (16) is equivalent to the following problem:

$$
\begin{array}{ll}
\max _{X} & \log \operatorname{det} X-\langle\Sigma, X\rangle-\rho e^{T}|X| e \\
\text { s.t. } & \alpha I \preceq X \preceq \beta I,
\end{array}
$$

for some $0<\alpha<\beta<\infty$. 
We further observe that problem (26) can be rewritten as

$$
\max _{X \in \mathcal{X}} \min _{U \in \mathcal{U}} \log \operatorname{det} X-\langle\Sigma+\rho U, X\rangle
$$

where $\mathcal{U}$ is defined in (19), and $\mathcal{X}$ is defined as follows:

$$
\mathcal{X}:=\left\{X \in \mathcal{S}^{n}: \alpha I \preceq X \preceq \beta I\right\} .
$$

Therefore, we conclude that problem (16) is equivalent to (27). For the remaining paper, we will focus on problem (27) only.

\subsection{Smooth optimization method for sparse covariance selection}

In this subsection, we describe the implementation details of the Smooth Minimization Algorithm proposed in Section 2 for solving problem (27). We also compare the complexity of this algorithm with interior point methods, and two other first-order methods studied in d'Aspremont et al. [9], that is, Nesterov's smooth approximation scheme and block coordinate descent method.

We first observe that the sets $\mathcal{X}$ and $\mathcal{U}$ both lie in the space $\mathcal{S}^{n}$, where $\mathcal{X}$ and $\mathcal{U}$ are defined in (28) and (19), respectively. Let $\mathcal{S}^{n}$ be endowed with the Frobenius norm, and let $\tilde{d}(X)=\log \operatorname{det} X$ for $X \in \mathcal{X}$. Then for any $X \in \mathcal{X}$, we have

$$
\nabla^{2} \tilde{d}(X)[H, H]=-\operatorname{Tr}\left(X^{-1} H X^{-1} H\right) \leq-\beta^{-2}\|H\|_{F}^{2}
$$

for all $H \in \mathcal{S}^{n}$, and hence, $\tilde{d}(X)$ is strongly concave on $\mathcal{X}$ with modulus $\beta^{-2}$. Using this result and Theorem 1 of [21, we immediately conclude that $\nabla f(U)$ is Lipschitz continuous with constant $L=\rho^{2} \beta^{2}$ on $\mathcal{U}$, where

$$
f(U):=\max _{X \in \mathcal{X}} \log \operatorname{det} X-\langle\Sigma+\rho U, X\rangle, \quad \forall U \in \mathcal{U} .
$$

Denote the unique optimal solution of problem (29) by $X(U)$. For any $U \in \mathcal{U}$, we can compute $X(U), f(U)$ and $\nabla f(U)$ as follows.

Let $\Sigma+\rho U=Q \operatorname{diag}(\gamma) Q^{T}$ be an eigenvalue decomposition of $\Sigma+\rho U$ such that $Q Q^{T}=I$. For $i=1, \ldots, n$, let

$$
\lambda_{i}= \begin{cases}\min \left\{\max \left\{1 / \gamma_{i}, \alpha\right\}, \beta\right\}, & \text { if } \gamma_{i}>0 \\ \beta, & \text { otherwise }\end{cases}
$$

It is not hard to show that

$$
X(U)=Q \operatorname{diag}(\lambda) Q^{T}, \quad f(U)=-\gamma^{T} \lambda+\sum_{i=1}^{n} \log \lambda_{i}, \quad \nabla f(U)=-\rho X(U) .
$$

From the above discussion, we see that problem (27) has exactly the same form as (2), and also satisfies all assumptions imposed on problem (2). Therefore, it can be suitably solved by 
the Smooth Minimization Algorithm proposed in Section 2. The implementation details of this algorithm for problem (27) are described as follows.

Given $U_{0} \in \mathcal{U}$, let $d(U)=\left\|U-U_{0}\right\|_{F}^{2} / 2$ be the proximal function on $\mathcal{U}$, which is strongly convex function with modulus $\sigma=1$. For our specific choice of the norm and $d(U)$, we clearly see that steps 2) and 3) of the Smooth Minimization Algorithm can be solved as a problem of the form

$$
V=\arg \min _{U \in \mathcal{U}}\langle G, U\rangle+\|U\|_{F}^{2} / 2
$$

for some $G \in \mathcal{S}^{n}$. In view of (19), we see that

$$
V_{i j}=\max \left\{\min \left\{-G_{i j}, 1\right\},-1\right\}, i, j=1, \ldots, n .
$$

In addition, for any $X \in \mathcal{X}$, we define

$$
g(X):=\log \operatorname{det} X-\langle\Sigma, X\rangle-\rho e^{T}|X| e .
$$

For the ease of comparison with its latter variant, we now present a complete version of the aforementioned Smooth Minimization Algorithm for solving problem (27) and its dual.

\section{Smooth Minimization Algorithm for Covariance Selection (SMACS):}

Let $\epsilon>0$ and $U_{0} \in \mathcal{U}$ be given. Set $X_{-1}=0, L=\rho^{2} \beta^{2}, \sigma=1$, and $k=0$.

1) Compute $\nabla f\left(U_{k}\right)$ and $X\left(U_{k}\right)$. Set $X_{k}=\frac{k}{k+2} X_{k-1}+\frac{2}{k+2} X\left(U_{k}\right)$.

2) Find $U_{k}^{s d}=\operatorname{argmin}\left\{\left\langle\nabla f\left(U_{k}\right), U-U_{k}\right\rangle+\frac{L}{2}\left\|U-U_{k}\right\|_{F}^{2}: U \in \mathcal{U}\right\}$.

3) Find $U_{k}^{a g}=\operatorname{argmin}\left\{\frac{L}{2 \sigma}\left\|U-U_{0}\right\|_{F}^{2}+\sum_{i=0}^{k} \frac{i+1}{2}\left[f\left(U_{i}\right)+\left\langle\nabla f\left(U_{i}\right), U-U_{i}\right\rangle\right]: U \in \mathcal{U}\right\}$.

4) Set $U_{k+1}=\frac{2}{k+3} U_{k}^{a g}+\frac{k+1}{k+3} U_{k}^{s d}$.

5) Set $k \leftarrow k+1$. Go to step 1) until $f\left(U_{k}^{s d}\right)-g\left(X_{k}\right) \leq \epsilon$.

\section{end}

The iteration complexity of the above algorithm for solving problem (27) is established in the following theorem.

Theorem 3.2 The iteration complexity performed by the algorithm SMACS for finding an $\epsilon$-optimal solution to problem (27) and its dual does not exceed $\sqrt{2} \rho \beta \max _{U \in \mathcal{U}}\left\|U-U_{0}\right\|_{F} / \sqrt{\epsilon}$, and moreover, if $U_{0}=0$, it does not exceed $\sqrt{2} \rho \beta n / \sqrt{\epsilon}$.

Proof. From the above discussion, we know that $L=\rho^{2} \beta^{2}, D=\max _{U \in \mathcal{U}}\left\|U-U_{0}\right\|_{F}^{2} / 2$ and $\sigma=1$, which together with Theorem 2.2 immediately implies that the first part of the 
statement holds. Further, if $U_{0}=0$, we easily obtain from (19) that $D=\max _{U \in \mathcal{U}}\|U\|_{F}^{2} / 2=n^{2} / 2$. The second part of the statement directly follows from this result and Theorem 2.2.

Remark. By the definition of $\mathcal{U}$ (see (19)), we can easily show that $\min _{U_{0} \in \mathcal{U}} \max _{U \in \mathcal{U}}\left\|U-U_{0}\right\|_{F}$ has a unique minimizer $U_{0}=0$. This result together with Theorem 3.2 implies that the initial point $U_{0}=0$ gives the optimal worst-case iteration complexity for the algorithm SMACS.

Alternatively, d'Aspremont et al. [9] applied Nesterov's smooth approximation scheme [21] to solve problem (27). More specifically, let $\epsilon>0$ be the desired accuracy, and let

$$
\hat{d}(U)=\|U\|_{F}^{2} / 2, \quad \hat{D}=\max _{U \in \mathcal{U}} \hat{d}(U)=n^{2} / 2 .
$$

As shown in [21], the non-smooth function $g(X)$ defined in (31) is uniformly approximated by the smooth function

$$
g_{\epsilon}(X)=\min _{U \in \mathcal{U}} \log \operatorname{det} X-\langle\Sigma+\rho U, X\rangle-\frac{\epsilon}{2 \hat{D}} \hat{d}(U)
$$

on $\mathcal{X}$ with the error at most by $\epsilon / 2$, and moreover, the function $g_{\epsilon}(X)$ has a Lipschitz continuous gradient on $\mathcal{X}$ with some constant $L(\epsilon)>0$. Nesterov's smooth optimization technique [19, 21] is then applied to solve the perturbed problem $\max _{X \in \mathcal{X}} g_{\epsilon}(X)$, and problem (27) is accordingly solved. It was shown in [9] that the iteration complexity of this approach for finding an $\epsilon$-optimal solution to problem (27) does not exceed

$$
\frac{2 \sqrt{2} \rho \beta n^{1.5} \log \kappa}{\epsilon}+\kappa \sqrt{\frac{n \log \kappa}{\epsilon}}
$$

where $\kappa:=\beta / \alpha$.

In view of (32) and Theorem 3.2, we conclude that the smooth optimization approach improves upon Nesterov's smooth approximation scheme at least by a factor $\mathcal{O}(\sqrt{n} \log \kappa / \sqrt{\epsilon})$ in terms of the iteration complexity for solving problem (27). Moreover, the computational cost per iteration of the former approach is at least as cheap as that of the latter one.

d'Aspremont et al. [9] also studied a block-coordinate descent method for solving problem (16) with $\tilde{\alpha}=0$ and $\tilde{\beta}=\infty$. Each iterate of this method requires computing the inverse of an $(n-1) \times(n-1)$ matrix, and solving a box constrained quadratic programming with $n-1$ variables. As mentioned in Section 3 of [9], this method has a local linear convergence rate. However, its global iteration complexity for finding an $\epsilon$-optimal solution is theoretically unknown. Moreover, this method is not suitable for solving problem (16) with $\tilde{\alpha}>0$ or $\tilde{\beta}<\infty$.

In addition, we observe that problem (26) (also (16)) can be reformulated as a constrained smooth convex problem that has an explicit $\mathcal{O}\left(n^{2}\right)$-logarithmically homogeneous selfconcordant barrier function. Thus, it can be suitably solved by interior point (IP) methods (see Nesterov and Nemirovski [22] and Vandenberghe et al. [24]). The worst-case iteration complexity of IP methods for finding an $\epsilon$-optimal solution to (26) is $\mathcal{O}\left(n \log \left(\epsilon_{0} / \epsilon\right)\right)$, where $\epsilon_{0}$ 
is an initial gap. Each iterate of IP methods requires $\mathcal{O}\left(n^{6}\right)$ arithmetic cost for assembling and solving a typically dense Newton system with $\mathcal{O}\left(n^{2}\right)$ variables. Thus, the total worst-case arithmetic cost of IP methods for finding an $\epsilon$-optimal solution to (26) is $\mathcal{O}\left(n^{7} \log \left(\epsilon_{0} / \epsilon\right)\right)$. In contrast with IP methods, the algorithm SMACS requires $\mathcal{O}\left(n^{3}\right)$ arithmetic cost per iteration dominated by eigenvalue decomposition and matrix multiplication of $n \times n$ matrices. Based on this observation and Theorem $[3.2$, we conclude that the overall worst-case arithmetic cost of the algorithm SMACS for finding an $\epsilon$-optimal solution to $(26)$ is $\mathcal{O}\left(\rho \beta n^{4} / \sqrt{\epsilon}\right)$, which is substantially superior to that of IP methods, provided that $\rho \beta$ is not too large and $\epsilon$ is not too small.

\subsection{Variant of Smooth Minimization Algorithm}

As discussed in Subsection 3.3, the algorithm SMACS has a nice theoretical complexity in contrast with IP methods, Nesterov's smooth approximation scheme, and block-coordinate descent method. However, its practical performance is still not much attractive (see Section 4). To enhance the computational performance, we propose a variant of the algorithm SMACS for solving problem (27) in this subsection.

Our first concern of the algorithm SMACS is that the eigenvalue decomposition of two $n \times n$ matrices is required per iteration. Indeed, the eigenvalue decomposition of $\Sigma+\rho U_{k}$ and $\Sigma+\rho U_{k}^{s d}$ is needed at steps 1$)$ and 5) to compute $\nabla f\left(U_{k}\right)$ and $f\left(U_{k}^{s d}\right)$, respectively. We also know that the eigenvalue decomposition is one of major computations for the algorithm SMACS. To reduce the computational cost, we now propose a new termination criterion other than $f\left(U_{k}^{s d}\right)-g\left(X_{k}\right) \leq \epsilon$ that is used in the algorithm SMACS. In view of Theorem 2.4, we know that

$$
f\left(U_{k}\right)-g\left(X\left(U_{k}\right)\right) \rightarrow 0, \text { as } k \rightarrow \infty .
$$

Thus, $f\left(U_{k}\right)-g\left(X\left(U_{k}\right)\right) \leq \epsilon$ can be used as an alternative termination criterion. Moreover, it follows from (30) that the quantity $f\left(U_{k}\right)-g\left(X\left(U_{k}\right)\right)$ is readily available in step 1) of the algorithm SMACS with almost no additional cost. We easily see that the algorithm SMACS with this new termination criterion would require only one eigenvalue decomposition per iteration. Despite this clear advantage, we shall mention that the iteration complexity of the resulting algorithm is unfortunately unknown. Nevertheless, in practice we have found that the number of iterations performed by the algorithm SMACS with the above two different termination criteria are almost same. Thus, $f\left(u_{k}\right)-g\left(x\left(u_{k}\right)\right) \leq \epsilon$ is a useful practical termination criterion.

For sparse covariance selection, the penalty parameter $\rho$ is usually small, but the parameter $\beta$ can be fairly large. In view of Theorem 3.2, we know that the iteration complexity of the algorithm SMACS for solving problem (27) is proportional to $\beta$. Therefore, when $\beta$ is too large, the complexity and practical performance of this algorithm become unattractive. To overcome this drawback, we will propose one strategy to dynamically update $\beta$.

Let $X^{*}$ be the unique optimal solution of problem (27). For any $\hat{\beta} \in\left[\lambda_{\max }\left(X^{*}\right), \beta\right]$, we easily observe that $X^{*}$ is also the unique optimal solution to the following problem:

$$
\left(P_{\hat{\beta}}\right) \quad \max _{X \in \mathcal{X}_{\hat{\beta}}} \min _{U \in \mathcal{U}} \log \operatorname{det} X-\langle\Sigma+\rho U, X\rangle,
$$


where $\mathcal{U}$ is defined in (19), and $\mathcal{X}_{\hat{\beta}}$ is given by

$$
\mathcal{X}_{\hat{\beta}}:=\{X: \alpha I \preceq X \preceq \hat{\beta} I\} .
$$

In view of Theorem [3.2, the iteration complexity of the algorithm SMACS for problem (33) is lower than that for problem (27) provided $\hat{\beta} \in\left[\lambda_{\max }\left(X^{*}\right), \beta\right)$. Hence ideally, we set $\hat{\beta}=$ $\lambda_{\max }\left(X^{*}\right)$, which would give the lowest iteration complexity, but unfortunately, $\lambda_{\max }\left(X^{*}\right)$ is unknown. However, we can generate a sequence $\left\{\hat{\beta}_{k}\right\}_{k=0}^{\infty}$ that asymptotically approaches $\lambda_{\max }\left(X^{*}\right)$ as the algorithm progresses. Indeed, in view of Theorem 2.4, we know that $X\left(U_{k}\right) \rightarrow$ $X^{*}$ as $k \rightarrow \infty$, and we obtain that

$$
\lambda_{\max }\left(X\left(U_{k}\right)\right) \rightarrow \lambda_{\max }\left(X^{*}\right), \text { as } k \rightarrow \infty .
$$

Therefore, we see that $\left\{\lambda_{\max }\left(X\left(U_{k}\right)\right)\right\}_{k=0}^{\infty}$ can be used to generate a sequence $\left\{\hat{\beta}_{k}\right\}_{k=0}^{\infty}$ that asymptotically approaches $\lambda_{\max }\left(X^{*}\right)$. We next propose a strategy to generate such a sequence $\left\{\hat{\beta}_{k}\right\}_{k=0}^{\infty}$.

For convenience of presentation, we introduce some new notations. Given any $U \in \mathcal{U}$ and $\hat{\beta} \in[\alpha, \beta]$, we define

$$
\begin{aligned}
X_{\hat{\beta}}(U) & :=\arg \max _{X \in \mathcal{X}_{\hat{\beta}}} \log \operatorname{det} X-\langle\Sigma+\rho U, X\rangle, \\
f_{\hat{\beta}}(U) & :=\max _{X \in \mathcal{X}_{\hat{\beta}}} \log \operatorname{det} X-\langle\Sigma+\rho U, X\rangle .
\end{aligned}
$$

Definition 1 Given any $U \in \mathcal{U}$ and $\hat{\beta} \in[\alpha, \beta], X_{\hat{\beta}}(U)$ is called "active" if $\lambda_{\max }\left(X_{\hat{\beta}}(U)\right)=\hat{\beta}$ and $\hat{\beta}<\beta$; otherwise it is called "inactive".

Let $\varsigma_{1}, \varsigma_{2}>1$, and $\varsigma_{3} \in(0,1)$ be given and fixed. Assume that $U_{k} \in \mathcal{U}$ and $\hat{\beta}_{k} \in[\alpha, \beta]$ are given at the beginning of the $k$ th iteration for some $k \geq 0$. We now describe the strategy for generating the next iterate $U_{k+1}$ and $\hat{\beta}_{k+1}$ by considering the following three different cases:

1) If $X_{\hat{\beta}_{k}}\left(U_{k}\right)$ is active, find the smallest $s \in \mathcal{Z}_{+}$such that $X_{\bar{\beta}}\left(U_{k}\right)$ is inactive, where $\bar{\beta}=\min \left\{\varsigma_{1}^{s} \hat{\beta}_{k}, \beta\right\}$. Set $\hat{\beta}_{k+1}=\bar{\beta}$, and apply the algorithm SMACS for problem $\left(P_{\hat{\beta}_{k+1}}\right)$ starting with the point $U_{k}$ and set its next iterate to be $U_{k+1}$.

2) If $X_{\hat{\beta}_{k}}\left(U_{k}\right)$ is inactive and $\lambda_{\max }\left(X_{\hat{\beta}_{k}}\left(U_{k}\right)\right) \leq \varsigma_{3} \hat{\beta}_{k}$, set $\hat{\beta}_{k+1}=\max \left\{\min \left\{\varsigma_{2} \lambda_{\max }\left(X_{\hat{\beta}_{k}}\left(U_{k}\right)\right)\right.\right.$, $\beta\}, \alpha\}$. Apply the algorithm SMACS for problem $\left(P_{\hat{\beta}_{k+1}}\right)$ starting with the point $U_{k}$, and set its next iterate to be $U_{k+1}$.

3) If $X_{\hat{\beta}_{k}}\left(U_{k}\right)$ is inactive and $\lambda_{\max }\left(X_{\hat{\beta}_{k}}\left(U_{k}\right)\right)>\varsigma_{3} \hat{\beta}_{k}$, set $\hat{\beta}_{k+1}=\hat{\beta}_{k}$. Continue the algorithm SMACS for problem $\left(P_{\hat{\beta}_{k}}\right)$, and set its next iterate to be $U_{k+1}$.

For the sequences $\left\{U_{k}\right\}_{k=0}^{\infty}$ and $\left\{\hat{\beta}_{k}\right\}_{k=0}^{\infty}$ recursively generated above, we observe that the sequence $\left\{X_{\hat{\beta}_{k+1}}\left(U_{k}\right)\right\}_{k=0}^{\infty}$ is always inactive. This together with (34), (35), (29) and the fact that $\hat{\beta}_{k} \leq \beta$ for $k \geq 0$, implies that

$$
f\left(U_{k}\right)=f_{\hat{\beta}_{k+1}}\left(U_{k}\right), \quad \nabla f\left(U_{k}\right)=\nabla f_{\hat{\beta}_{k+1}}\left(U_{k}\right), \quad \forall k \geq 0 .
$$


Therefore, the new termination criterion $f\left(U_{k}\right)-g\left(X\left(U_{k}\right)\right) \leq \epsilon$ can be replaced by

$$
f_{\hat{\beta}_{k+1}}\left(U_{k}\right)-g\left(X_{\hat{\beta}_{k+1}}\left(U_{k}\right)\right) \leq \epsilon
$$

accordingly.

We now incorporate into the algorithm SMACS the new termination criterion (37) and the aforementioned strategy for generating a sequence $\left\{\hat{\beta}_{k}\right\}_{k=0}^{\infty}$ that asymptotically approaches $\lambda_{\max }\left(X^{*}\right)$, and obtain a variant of the algorithm SMACS for solving problem (27). For convenience of presentation, we omit the subscript $k$ from $\hat{\beta}_{k}$.

Variant of Smooth Minimization Algorithm for Covariance Selection (VSMACS):

Let $\epsilon>0, \varsigma_{1}, \varsigma_{2}>1$, and $\varsigma_{3} \in(0,1)$ be given. Choose a $U_{0} \in \mathcal{U}$. Set $\hat{\beta}=\beta, L=\rho^{2} \beta^{2}, \sigma=1$, and $k=0$.

1) Compute $X_{\hat{\beta}}\left(U_{k}\right)$ according to (30).

1a) If $X_{\hat{\beta}}\left(U_{k}\right)$ is active, find the smallest $s \in \mathcal{Z}_{+}$such that $X_{\bar{\beta}}\left(U_{k}\right)$ is inactive, where $\bar{\beta}=\min \left\{\varsigma_{1}^{s} \hat{\beta}, \beta\right\}$. Set $k=0, U_{0}=U_{k}, \hat{\beta}=\bar{\beta}, L=\rho^{2} \hat{\beta}^{2}$, and go to step 2).

1b) If $X_{\hat{\beta}}\left(U_{k}\right)$ is inactive and $\lambda_{\max }\left(X_{\hat{\beta}}\left(U_{k}\right)\right) \leq \varsigma_{3} \hat{\beta}$, set $k=0, U_{0}=U_{k}$, $\hat{\beta}=\max \left\{\min \left\{\varsigma_{2} \lambda_{\max }\left(X_{\hat{\beta}}\left(U_{k}\right)\right), \beta\right\}, \alpha\right\}$, and $L=\rho^{2} \hat{\beta}^{2}$.

2) If $f_{\hat{\beta}}\left(U_{k}\right)-g\left(X_{\hat{\beta}}\left(U_{k}\right)\right) \leq \epsilon$, terminate. Otherwise, compute $\nabla f_{\hat{\beta}}\left(U_{k}\right)$ according to (30).

3) Find $U_{k}^{s d}=\operatorname{argmin}\left\{\left\langle\nabla f_{\hat{\beta}}\left(U_{k}\right), U-U_{k}\right\rangle+\frac{L}{2}\left\|U-U_{k}\right\|_{F}^{2}: U \in \mathcal{U}\right\}$.

4) Find $U_{k}^{a g}=\operatorname{argmin}\left\{\frac{L}{2 \sigma}\left\|U-U_{0}\right\|_{F}^{2}+\sum_{i=0}^{k} \frac{i+1}{2}\left[f_{\hat{\beta}}\left(U_{i}\right)+\left\langle\nabla f_{\hat{\beta}}\left(U_{i}\right), U-U_{i}\right\rangle\right]: U \in \mathcal{U}\right\}$.

5) Set $U_{k+1}=\frac{2}{k+3} U_{k}^{a g}+\frac{k+1}{k+3} U_{k}^{s d}$.

6) Set $k \leftarrow k+1$, and go to step 1).

end

We next establish some preliminary convergence properties of the above algorithm.

Proposition 3.3 For the algorithm VSMACS, the following properties hold:

1) Suppose that the algorithm VSMACS terminates at some iterate $\left(X_{\hat{\beta}}\left(U_{k}\right), U_{k}\right)$. Then $\left(X_{\hat{\beta}}\left(U_{k}\right), U_{k}\right)$ is an $\epsilon$-optimal solution to problem (27) and its dual.

2) Suppose that $\hat{\beta}$ is updated only for a finite number of times. Then the algorithm VSMACS terminates in a finite number of iterations, and produces an $\epsilon$-optimal solution to problem (27) and its dual. 
Proof. For the final iterate $\left(X_{\hat{\beta}}\left(U_{k}\right), U_{k}\right)$, we clearly know that $f_{\hat{\beta}}\left(U_{k}\right)-g\left(X_{\hat{\beta}}\left(U_{k}\right)\right) \leq \epsilon$, and $X_{\hat{\beta}}\left(U_{k}\right)$ is inactive. As shown in (36), $f\left(U_{k}\right)=f_{\hat{\beta}}\left(U_{k}\right)$. Hence, we have $f\left(U_{k}\right)-g\left(X_{\hat{\beta}}\left(U_{k}\right)\right) \leq$ $\epsilon$. We also know that $U_{k} \in \mathcal{U}$, and $X_{\hat{\beta}}\left(U_{k}\right) \in \mathcal{X}$ due to $\hat{\beta} \in[\alpha, \beta]$. Thus, statement 1) immediately follows. After the last update of $\hat{\beta}$, the algorithm VSMACS behaves exactly like the algorithm SMACS as applied to solve problem $\left(P_{\hat{\beta}}\right)$ except with the termination criterion $f\left(U_{k}\right)-g\left(X_{\hat{\beta}}\left(U_{k}\right)\right) \leq \epsilon$. Thus, it follows from statement 1) and Theorem 2.4 that statement 2) holds.

\section{Computational results}

In this section, we compare the performance of the smooth minimization approach and its variant proposed in this paper with other first-order methods studied in [9, 15], that is, Nesterov's smooth approximation scheme and block coordinate descent method for solving problem (16) (or equivalently, (27)) on a set of randomly generated instances.

All instances used in this section were randomly generated in the same manner as described in d'Aspremont et al. [9]. First, we generate a sparse invertible matrix $A \in \mathcal{S}^{n}$ with positive diagonal entries and a density prescribed by $\varrho$. We then generate the matrix $B \in \mathcal{S}^{n}$ by

$$
B=A^{-1}+\tau V
$$

where $V \in \mathcal{S}^{n}$ is an independent and identically distributed uniform random matrix, and $\tau$ is a small positive number. Finally, we obtain the following randomly generated sample covariance matrix:

$$
\Sigma=B-\min \left\{\lambda_{\min }(B)-\vartheta, 0\right\} I,
$$

where $\vartheta$ is a small positive number. In particular, we set $\varrho=0.01, \tau=0.15$ and $\vartheta=1.0 e-4$ for generating all instances.

As discussed in Section 3.3, our smooth minimization approach has much better worst-case iteration complexity than Nesterov's smooth approximation scheme studied in d'Aspremont et al. [9] for problem (27). However, it is unknown how their practical performance differs from each other. In the first experiment, we compare the practical performance of our smooth minimization approach and its variant with Nesterov's smooth approximation scheme studied in d'Aspremont et al. [9] for problem (27) with $\alpha=0.1, \beta=10$ and $\rho=0.5$. For convenience of presentation, we label these three first-order methods as SM, VSM, and NSA, respectively. The codes for them are written in Matlab. More specifically, the code for NSA follows the algorithm presented in d'Aspremont et al. [9], and the codes for SM and VSM are written in accordance with the algorithms SMACS and VSMACS, respectively. Moreover, we set $\varsigma_{1}=\varsigma_{2}=1.05$ and $\varsigma_{3}=0.95$ for the algorithm VSMACS. These three methods terminate once the duality gap is less than $\epsilon=0.1$. All computations are performed on an Intel Xeon 2.66 $\mathrm{GHz}$ machine with Red Hat Linux version 8.

The performance of the methods NSA, SM and VSM for the randomly generated instances are presented in Table 1. The row size $n$ of each sample covariance matrix $\Sigma$ is given in column one. The numbers of iterations of NSA, SM and VSM are given in columns two to four, 
Table 1: Comparison of NSA, SM and VSM

\begin{tabular}{|c||rrr||rrr||rrr|}
\hline \multicolumn{1}{|c||}{$\begin{array}{c}\text { Problem } \\
\mathrm{n}\end{array}$} & \multicolumn{3}{c||}{ NSA } & \multicolumn{1}{c||}{ SM } & \multicolumn{1}{c||}{ VSM } & \multicolumn{1}{|c||}{ NSA } & \multicolumn{1}{c|}{ SM } & \multicolumn{1}{c|}{ VSM } & \multicolumn{3}{c|}{ NSA } & SM & VSM \\
\hline 50 & 3657 & 457 & 20 & -76.399 & -76.399 & -76.393 & 49.0 & 2.7 & 0.1 \\
100 & 7629 & 920 & 27 & -186.717 & -186.720 & -186.714 & 900.4 & 38.4 & 0.4 \\
150 & 20358 & 1455 & 49 & -318.195 & -318.194 & -318.184 & 8165.7 & 188.8 & 2.0 \\
200 & 27499 & 2294 & 102 & -511.246 & -511.245 & -511.242 & 26172.5 & 698.8 & 9.2 \\
250 & 45122 & 3060 & 128 & -3793.255 & -3793.256 & -3793.257 & 87298.9 & 1767.9 & 19.8 \\
300 & 54734 & 3881 & 161 & -3187.163 & -3187.171 & -3187.172 & 184798.1 & 3994.0 & 45.5 \\
350 & 64641 & 4634 & 182 & -2756.717 & -2756.734 & -2756.734 & 351460.7 & 7613.9 & 83.6 \\
400 & 74839 & 5308 & 176 & -3490.640 & -3490.667 & -3490.667 & 614237.1 & 13536.7 & 116.9 \\
\hline
\end{tabular}

and the objective function values are given in columns five to seven, and the CPU times (in seconds) are given in the last three columns, respectively. From Table 1, we conclude that: i) the method SM, namely, the smooth minimization approach, outperforms substantially the method NSA, that is, Nesterov's smooth approximation scheme; and ii) the method VSM, namely, the variant of the smooth minimization approach, substantially outperforms the other two methods. In addition, we see from this experiment that Nesterov's smooth minimization approach [19] is generally more appealing than his smooth approximation scheme [21] whenever the problem can be solved as an equivalent smooth problem. Nevertheless, we shall mention that the latter approach has much wider field of application (e.g., see [21]), where the former approach cannot be applied.

From the above experiment, we have already seen that the method VSM outperforms substantially two other first-order methods, namely, SM and NSA for solving problem (27). In the second experiment, we compare the performance of the method VSM with the block coordinate descent (BCD) methods studied in d'Aspremont et al. 9] and Friedman et al. [15] on relatively large-scale instances. For convenience of presentation, we label these two methods as BCD1 and BCD2, respectively. The method BCD2 was developed very recently and it is a slight variant of the method BCD1. In particular, each iterate of BCD1 solves a box constrained quadratic programming by means of interior point methods, but each iterate of BCD2 applies a coordinate descent approach to solving a lasso ( $l_{1}$-regularized) least-squares problem, which is the dual of the box constrained quadratic programming appearing in BCD1. It is worth mentioning the methods BCD1 and BCD2 are only applicable for solving problem (16) with $\tilde{\alpha}=0$ and $\tilde{\beta}=\infty$. Thus, we only compare their performance with our method VSM for problem (16) with such $\tilde{\alpha}$ and $\tilde{\beta}$. As shown in Subsection 3.2, problem (16) with $\tilde{\alpha}=0$ and $\tilde{\beta}=\infty$ is equivalent to problem (27) with $\alpha$ and $\beta$ given in (18), and hence it can be solved by applying the method VSM to the latter problem instead.

The code for the method BCD1 was written in Matlab by d'Aspremont and El Ghaoui [10] while the code for BCD2 was written in Fortran 90 by Friedman et al. [16. The methods BCD1 and VSM terminate once the duality gap is less than $\epsilon=0.1$. The original code [16] for BCD2 uses the average absolute change in the approximate solution as the termination criterion. In particular, the average absolute change in the approximate solution is evaluated at the end of 
Table 2: Comparison of BCD1, BCD2 and VSM

\begin{tabular}{|c||rrr||rrr||rrr|}
\hline \multicolumn{1}{|c||}{$\begin{array}{c}\text { Problem } \\
\mathrm{n}\end{array}$} & \multicolumn{3}{|c||}{ Iter } & \multicolumn{3}{c||}{ Obj } & \multicolumn{3}{c|}{ Time } \\
\hline 100 & 124 & 200 & 33 & -186.522 & -186.433 & -186.522 & 22.3 & 0.1 & 0.5 \\
200 & 531 & 600 & 109 & -449.210 & -449.179 & -449.209 & 300.0 & 1.3 & 9.5 \\
300 & 1530 & 1500 & 146 & -767.615 & -767.608 & -767.614 & 2428.2 & 80.9 & 48.5 \\
400 & 2259 & 2400 & 154 & -1082.679 & -1082.651 & -1082.677 & 8402.4 & 298.7 & 112.3 \\
500 & 3050 & 3500 & 154 & -1402.503 & -1402.457 & -1402.502 & 22537.1 & 640.2 & 211.5 \\
600 & 3705 & 4200 & 165 & -1728.628 & -1728.587 & -1728.627 & 48950.4 & 1215.0 & 397.6 \\
700 & 4492 & 4900 & 163 & -2057.894 & -2057.862 & -2057.892 & 92052.7 & 1972.5 & 611.1 \\
800 & 4958 & 5600 & 169 & -2392.713 & -2392.671 & -2392.712 & 147778.9 & 2872.3 & 943.2 \\
900 & 5697 & 6300 & 161 & -2711.874 & -2711.827 & -2711.874 & 219644.3 & 3593.7 & 1268.5 \\
1000 & 6536 & 7000 & 161 & -3045.808 & -3045.768 & -3045.808 & 344687.8 & 6098.7 & 1710.0 \\
\hline
\end{tabular}

each cycle consisting of $n$ block coordinate descent iterations, and their code terminates once it is below a given accuracy (see pp. 6 of [15] for details). According to our computational experience, we found with such a criterion, BCD2 is extremely hard to terminate for relatively large-scale instances (say $n=300$ ) unless a maximum number of iterations is set. Obviously, it is not easy to choose a suitable maximum number of iterations for BCD2. Thus, to be as fair as possible to BCD1 and VSM, we simply replace their termination criterion detailed in [16] for BCD2 by the one with the duality gap less than $\epsilon=0.1$. In other words, the duality gap is computed at the end of each cycle consisting of $n$ block coordinate descent iterations, and $\mathrm{BCD} 2$ terminates once it is below $\epsilon=0.1$. It is worth remarking that the cost for computing a duality gap is $\mathcal{O}\left(n^{3}\right)$ since the inverse of an $n \times n$ symmetric matrix is needed. Thus, it is reasonable to compute duality gap once every $n$ iterations rather than each iteration.

All computations are performed on an Intel Xeon $2.66 \mathrm{GHz}$ machine with Red Hat Linux version 8. The performance of the methods BCD1, BCD2 and VSM for the randomly generated instances are presented in Table 2, The row size $n$ of each sample covariance matrix $\Sigma$ is given in column one. The numbers of iterations of BCD1, BCD2 and VSM are given in columns two to four, and the objective function values are given in columns five to eight, and the CPU times (in seconds) are given in the last three columns, respectively. From Table 2. we conclude both BCD2 and VSM substantially outperform BCD1. We also observe that our method VSM outperforms BCD2 for almost all instances except two relatively small-scale instances.

In the above experimentation, we compared the performance of BCD2 and VSM for $\epsilon=0.1$. We next compare their performance on the same instances as above and apply the same termination criterion as above except that we set up $\epsilon=0.01$ and an upper bound of 20 hours computation time (or 72, 000 seconds) per instance for both codes. The performance of the methods BCD2 and VSM are presented in Table 3. The row size $n$ of each sample covariance matrix $\Sigma$ is given in column one. The numbers of iterations of BCD2 and VSM are given in columns two to three, and the objective function values are given in columns four to five, and the CPU times (in seconds) are given in the last two columns, respectively. It shall be 
Table 3: Comparison of BCD2 and VSM

\begin{tabular}{|c||rr||rr||rr|}
\hline \multicolumn{1}{|c||}{ Problem } & \multicolumn{2}{c||}{ Iter } & \multicolumn{2}{c||}{ Obj } & \multicolumn{2}{c|}{ Time } \\
$\mathrm{n}$ & BCD2 & VSM & BCD2 & VSM & BCD2 & VSM \\
\hline 100 & 200 & 54 & -186.433 & -186.435 & 0.1 & 0.77 \\
200 & 1200 & 239 & -449.119 & -449.122 & 2.1 & 21.6 \\
300 & 3000 & 310 & -767.525 & -767.525 & 32.1 & 104.2 \\
400 & 11778400 & 321 & -1082.592 & -1082.589 & 72000.0 & 223.3 \\
500 & 6997000 & 309 & -1402.420 & -1402.413 & 72001.0 & 395.5 \\
600 & 4637400 & 318 & -1728.553 & -1728.538 & 72004.0 & 765.2 \\
700 & 3215100 & 310 & -2057.823 & -2057.804 & 72005.0 & 1330.0 \\
800 & 2307200 & 309 & -2392.644 & -2392.623 & 72003.0 & 1789.2 \\
900 & 1846800 & 289 & -2711.806 & -2711.784 & 72024.0 & 2394.0 \\
1000 & 1257000 & 283 & -3045.749 & -3045.718 & 72051.0 & 3115.8 \\
\hline
\end{tabular}

mentioned that BCD2 and VSM are both feasible methods, and moreover, (16) and (27) are maximization problems. Therefore for these two methods, the larger objective function value, the better. From Table 3 , we observe that up to accuracy $\epsilon=0.01$, the method BCD2 cannot solve almost all instances within 20 hours except the first three relatively small-scale ones, but our method VSM does solve each of these instances in less than one hour and produces a better objective function values for almost all instances except the first three relatively small-scale ones. Also, it is interesting to observe that the number of iterations for VSM nearly doubles as the accuracy parameter $\epsilon$ increases by one digit, which is even better than the theoretical estimate that is $\sqrt{10}$ according to Theorem 3.2 .

\section{Concluding remarks}

In this paper, we proposed a smooth optimization approach for solving a class of non-smooth strictly concave maximization problems. We also discussed the application of this approach to sparse covariance selection, and proposed a variant of this approach. The computational results showed that the variant of the smooth optimization approach outperforms substantially the latter one, and two other first-order methods studied in d'Aspremont et al. 9] and Friedman et al. [15].

As discussed in Subsection 3.3. problem (27) has the same form as (2), and satisfies all assumptions imposed on problem (2). Moreover, its associated objective function $\phi(X, U)=$ $\log \operatorname{det} X-\langle\Sigma+\rho U, X\rangle$ is affine with respect to $U$ for every fixed $X \in \mathcal{S}_{++}^{n}$. In view of these facts along with the remarks made in Section 2, one can observe that problem (27) can be suitably solved by Nesterov's excessive gap technique [20]. Since the iterate complexity and the computational cost per iterate of this technique is same as those of the algorithm SMACS, we expect that the computational performance of these two methods for solving (27) are similar. It would be interesting to implement Nesterov's excessive gap technique [20] and its variant (that is, the one in a similar fashion to the algorithm VSMACS), and compare 
their computational performance with SMACS and VSMACS, respectively.

Though the variant of the smooth optimization approach outperforms substantially the smooth optimization approach, we are currently only able to establish some preliminary convergence properties for it. A possible direction leading to a thorough proof of its convergence would be to show that the updates on $\hat{\beta}$ in the algorithm VSMACS can occur only for a finite number of times. Given that VSMACS is a nonmonotone algorithm, it is, however, highly challenging to analyze the behavior of the sequences $\left\{U_{k}\right\}$ and $\left\{X_{\hat{\beta}}\left(U_{k}\right)\right\}$ and hence the total number of updates on $\hat{\beta}$. Interestingly, we observed in our implementation that when $\hat{\beta}>\lambda_{\max }\left(X^{*}\right)$, the sequence $\left\{X_{\hat{\beta}}\left(U_{k}\right)\right\}$ generated by the algorithm VSMACS satisfies $\lambda_{\max }\left(X_{\hat{\beta}}\left(U_{k}\right)\right) \in\left[\lambda_{\max }\left(X^{*}\right), \hat{\beta}\right)$, where $X^{*}$ is the optimal solution of problem (27). Nevertheless, it remains completely open whether this holds in general or not. In addition, the ideas used in the variant of the smooth optimization approach are interesting in their own right even when viewed as some heuristics. They could also be used to enhance the practical performance of Nesterov's first-order methods [19, 21] for solving some general min-max problems.

The codes for the variant of the smooth minimization approach are written in Matlab and C, which are available online at www.math.sfu.ca/ zhaosong. The C code for this method can solve large-scale problems more efficiently provided that LAPACK package is suitably installed. We will plan to extend these codes for solving more general problems of the form

$$
\begin{array}{cl}
\max _{X} & \log \operatorname{det} X-\langle\Sigma, X\rangle-\sum_{i j} \omega_{i j}\left|X_{i j}\right| \\
\text { s.t. } & \tilde{\alpha} I \preceq X \preceq \tilde{\beta} I, \\
& X_{i j}=0, \forall(i, j) \in \Omega,
\end{array}
$$

for some set $\Omega$, where $\omega_{i j}=\omega_{j i} \geq 0$ for all $i, j=1, \ldots, n$, and $0 \leq \tilde{\alpha}<\tilde{\beta} \leq \infty$ are some fixed bounds on the eigenvalues of the solution.

\section{Acknowledgement}

The author would like to thank Professor Alexandre d'Aspremont for a careful discussion on the iteration complexity of Nesterov's smooth approximation scheme for sparse covariance selection given in [9]. Also, the author is in debt to two anonymous referees for numerous insightful comments and suggestions, which have greatly improved the paper.

\section{References}

[1] J. Akaike. Information theory and an extension of the maximum likelihood principle. In B. N. Petrov and F. Csaki, editors, Second international symposium on information theory, pages 267-281. Akedemiai Kiado, Budapest, 1973.

[2] A. Auslender and M. Teboulle. Interior gradient and proximal methods for convex and conic optimization. SIAM Journal on Optimization, 16:697-725, 2006. 
[3] O. Banerjee, L. El Ghaoui, A. d'Aspremont, and G. Natsoulis. Convex optimization techniques for fitting sparse gaussian graphical models. In ICML '06: Proceedings of the 23rd international conference on Machine learning, pages 89-96, New York, NY, USA, 2006. ACM Press.

[4] J. A. Bilmes. Factored sparse inverse covariance matrices. Proceedings of IEEE International Conference on Acoustics, Speech, and Signal Processing, 2:1009-1012, 2000.

[5] K. P. Burnham and R. D. Anderson. Multimodel inference. understanding aic or bic in model selection. Sociological methods and research, 33:261-304, 2004.

[6] S. Chen, D. Donoho, and M. Saunders. Atomic decomposition by basis pursuit. SIAM Journal on Scientific Computing, 20:33-61, 1998.

[7] J. Dahl, V. Roychowdhury, and L. Vandenberghe. Maximum likelihood estimation of gaussian graphical models: numerical implementation and topology selection. Manuscript, University of California Los Angeles, October 2004.

[8] J. Dahl, L. Vandenberghe, and V. Roychowdhury. Covariance selection for non-chordal graphs via chordal embedding. Manuscript, University of California Los Angeles, July 2006. Submitted to Optimization Methods and Software.

[9] A. d'Aspremont, O. Banerjee, and L. El Ghaoui. First-order methods for sparse covariance selection. To appear in SIAM Journal on Matrix Analysis and Applications.

[10] A. d'Aspremont and L. El Ghaoui. Covsel: First order methods for sparse covariance selection, March 2006.

[11] A. Dempster. Covariance selection. Biometrics, 28:157-175, 1972.

[12] A. Dobra, C. Hans, B. Jones, J. R. Nevins, G. Yao, and M. West. Sparse graphical models for exploring gene expression data. Journal of Multivariate Analysis, 90:196-212, 2004.

[13] A. Dobra and M. West. Bayesian covariance selection. Isds working paper, Duke University, July 2004.

[14] D. L. Donoho and J. Tanner. Sparse nonnegative solutions of underdetermined linear equations by linear programming. Proceedings of the National Academy of Sciences, 102:9446-9451, 2005.

[15] J. Friedman, T. Hastie, and R. Tibshirani. Sparse inverse covariance estimation with the graphical lasso. Biostatistics, pages 1-10, December 2007.

[16] J. Friedman, T. Hastie, and R. Tibshirani. glasso: Graphical lasso for R, November 2007.

[17] J. Z. Huang, N. Liu, and M. Pourahmadi. Covariance matrix selection and estimation via penalised normal likelihood. Biometrika, 93(1):85-98, 2006. 
[18] B. Jones, C. Carvalho, C. Dobra, A. Hans, C. Carter, and M. West. Experiments in stochastic computation for high-dimensional graphical models. Statistical Science, 20:388-400, 2005.

[19] Y. E. Nesterov. A method for unconstrained convex minimization problem with the rate of convergence $O\left(1 / k^{2}\right)$. Doklady AN SSSR, 269:543-547, 1983. translated as Soviet Math. Docl.

[20] Y. E. Nesterov. Excessive gap technique in nonsmooth convex minimization. SIAM Journal on Optimization, 16:235-249, 2005.

[21] Y. E. Nesterov. Smooth minimization of nonsmooth functions. Mathematical Programming, 103:127-152, 2005.

[22] Y. E. Nesterov and A. S. Nemirovski. Interior point Polynomial algorithms in Convex Programming: Theory and Applications. Society for Industrial and Applied Mathematics, Philadelphia, 1994.

[23] R. Tibshirani. Regression shrinkage and selection via the lasso. Journal of the Royal statistical society, series B, 58:267-288, 1996.

[24] L. Vandenberghe, S. Boyd, and S. Wu. Determinant maximization with linear matrix inequality constraints. SIAM Journal on Matrix Analysis and Applications, 19(2):499$533,1998$.

[25] M. Yuan and Y. Lin. Model selection and estimation in the Gaussian graphical model. Biometrika, 94(1):19-35, 2007. 